\title{
Comparison of canonical bases for Schur and universal enveloping algebras
}

\author{
Ben Webster' \\ Department of Mathematics, University of Virginia, Charlottesville, VA
}

\begin{abstract}
We show that the canonical bases in $\dot{U}\left(\mathfrak{s I}_{n}\right)$ and the Schur algebra are compatible; in fact we extend this result to $p$-canonical bases. This follows immediately from a fullness result for a functor categorifying this map. In order to prove this result, we also explain the connections between categorifications of the Schur algebra which arise from parity sheaves on partial flag varieties, singular Soergel bimodules and Khovanov and Lauda's "flag category," which are of some independent interest.
\end{abstract}

\section{INTRODUCTION}

Numerous algebras and representations that appear in Lie theory have bases which are called "canonical." There are a variety of arguments for the importance of these bases, but surely one of their most desirable properties is that these bases match under natural maps of algebras.

The example we'll consider in this paper is the natural projection $\phi: U_{q}\left(\mathfrak{s l}_{n}\right) \rightarrow$ $S_{q}(d, n)$ from the quantized universal enveloping algebra to the $q$-Schur algebra. The latter algebra actually appears more naturally as the modified quantum group $\mathbf{U}$ with idempotents $1_{\lambda}$ for the different weights $\lambda$ added. In this case, the Schur algebra $S_{q}(d, n)$ can be thought of as the quotient of $\dot{U}$ which kills $1_{\lambda}$ is $\lambda$ cannot be written in the form $\lambda=\left(a_{1}, \ldots, a_{n}\right)$ with $\sum_{i} a_{i}=d$ and $a_{i} \geq 0$.

The algebra $\dot{U}$ is endowed with a canonical basis $\dot{\mathbf{B}}$ defined by Lusztig [Lus93], and the Schur algebra $S_{q}(d, n)$ with a canonical basis $\dot{\mathbf{B}}_{d}$ (also called IC basis) given by realizing it as the algebra of $G L_{d}\left(\mathbb{F}_{q}\right)$-invariant functions on the space of pairs of flags of length $n$ (of all possible dimensions) in the space $\mathbb{F}_{q}^{d}$, and considering the functions attached the IC sheaves smooth along $G L_{d}\left(\mathbb{F}_{p}\right)$-orbits [BLM90].

Theorem A. Under the map $\phi$, the set $\dot{\mathbf{B}} \backslash(\dot{\mathbf{B}} \cap \operatorname{ker} \phi)$ is sent bijectively to $\dot{\mathbf{B}}_{d}$.

It's worth noting that this theorem was proven in [SV00]. However, there it is submerged as a special case of a more general theorem, and new techniques have appeared in the literature in the time since that paper that allow us to give a more straightforward and modern proof. Key among these is the notion of a categorical action introduced by Rouquier [Rou] and Khovanov and Lauda [KL10]. We also give a generalization of this result to include $p$-canonical bases, which proceeds along the same lines. We also include several results which while familiar-sounding to an expert reader seem not to have made a clear appearance in the literature.

\footnotetext{
${ }^{1}$ Supported by the NSF under Grant DMS-1151473
} 
The map $\phi$ has a categorification which is well-established in the literature (though its connection to the Schur algebra is perhaps less well-known). For simplicity in the introduction, we'll only use categorifications of characteristic 0 .

- The algebra $\dot{U}$ is the Grothendieck group of a 2-category $\mathcal{U}$ defined in Rouquier [Rou] and Khovanov and Lauda [KL10] (these definitions were recently shown to be equivalent by Brundan [Bru]]). Actually, we'll use a slightly modified category $U$ with the same Grothendieck group. The indecomposable 1-morphisms correspond to the canonical basis B by [Web15, Th. A].

- The algebra $S_{q}(d, n)$ is categorified by Khovanov and Lauda's flag category Flag $_{d}[$ KL10, §5.3]. The indecomposable 1-morphisms correspond to the canonical basis $\dot{\mathbf{B}}_{d}$, a fact we'll establish later.

- The map $\phi$ is categorified by a 2 -functor $\Phi: \mathcal{U} \rightarrow$ Flag $_{d}$ defined by Khovanov and Lauda.

This categorical perspective helps to show the match of the bases above; it follows immediately from the fact that functor above sends indecomposable objects to indecomposable objects, itself a consequence of:

Theorem B. The functor $\Phi$ is full on 2-morphisms, i.e., it induces a surjective map $\operatorname{Hom}_{\mathcal{U}}(u, v) \rightarrow \operatorname{Hom}_{\text {Flag }_{d}}(\Phi u, \Phi v)$ for all 1-morphisms $u, v$.

Besides its interesting consequence for canonical bases, this also a beautiful illustration of the power of a categorical approach. While it may be that a direct proof of Theorem $\mathrm{A}$ on the level of Schur algebras exists, the author has had no luck in finding one.

It's also worth noting that a similar fullness result is proven for $\mathfrak{s l}_{2}$ by Beliakova and Lauda in [BL14]. We will briefly indicate how their result generalizes in this case.

\section{BACKGROUND}

Throughout, we'll fix a field $\mathbf{k}$ which may be of any characteristic.

2.1. Categorified $\mathfrak{s l}_{n}$. One of the basic objects we'll consider is the 2-category $\mathcal{U}$ categorifying $\dot{U}$. Rather than give a full definition of this category, we refer the reader to a number of papers which give this definition, such as [KL10, Rou, CL15, Web]. We will follow the conventions of [KL10] for simplicity, with the single exception that we think of the objects as elements of $\mathbb{Z}^{n}$ (i.e. the $\mathrm{gI}_{n}$ weight lattice) rather than $\mathbb{Z}^{n} / \mathbb{Z} \cdot(1, \ldots, 1)$, the weight lattice of $\mathfrak{s l}_{n}$. As we mentioned earlier, work of [Bru] shows that while the definitions given in other papers may not immediately look equivalent, they in fact are. The important points of the definition are that $\mathcal{U}$ is a graded strict 2-category with:

- objects given by the weight lattice of $\mathfrak{g l}_{n}$, given by $\mathbb{Z}^{n}$. In this space there are distinguished elements $\alpha_{i}=(0, \ldots, 1,-1, \ldots 0)$.

- 1-morphisms freely generated by symbols $\mathcal{E}_{i}: \lambda \rightarrow \lambda+\alpha_{i}$ and $\mathcal{F}_{i}: \lambda \rightarrow \lambda-\alpha_{i}$. Note that if two vectors do not have the same sum of their entries, then there are no 1-morphisms linking them. 
- 2-morphisms given by certain diagrams which induce a biadjunction between $\mathcal{F}_{i}$ and $\mathcal{E}_{i}$ (up to grading shift), and an isomorphism (up to grading shift)

$$
\operatorname{id}_{\mu}^{\mu_{i+1}} \oplus \mathcal{E}_{i} \mathcal{F}_{i} \cong \mathcal{F}_{i} \mathcal{E}_{i} \oplus \mathrm{id}_{\mu}^{\mu_{i}}
$$

where $\mu=\left(\mu_{1}, \ldots, \mu_{n}\right)$.

Remark 1. As mentioned above, this is a slight variation on Khovanov and Lauda's category; if we consider weights whose entries have a fixed sum d, this is equivalent to Khovanov and Lauda's category for weights where the entries sum to $d(\bmod n)$ (in the weight lattice of $\mathfrak{s l}_{n}$, only this residue is well defined) via the natural map $\mathbb{Z}^{n} \rightarrow \mathbb{Z}^{n} / \mathbb{Z} \cdot\langle(1, \ldots, 1)$.

This category carries a duality functor defined in [KL10, §3.3.2] such that $\mathcal{E}_{i}$ and $\mathcal{F}_{i}$ are self-dual. As mentioned in the introduction, this serves as a categorical avatar of the canonical basis.

Theorem 2 ([Web15, Th. A]). There is an isomorphism $K(\mathcal{U}) \cong U_{q}\left(\mathfrak{s l}_{n}\right)$. If $\mathbf{k}$ has characteristic 0 , then the indecomposable self-dual 1-morphisms in $\mathcal{U}$ match the basis $\dot{\mathbf{B}}$.

For a general field $\mathbf{k}$, the classes of the self-dual indecomposables only depend on the characteristic of the field by [Web15, 5.11]; the indecomposables with $\mathbf{k}=\mathbb{F}_{p}$ give a new basis $\dot{\mathbf{B}}^{(p)}$, usually called $p$-canonical or orthodox; this will agree with the usual canonical basis for $p$ large.

It will be useful for us to consider a slightly larger category; there is a natural inclusion of $\mathcal{U} \rightarrow \mathcal{U}_{\mathfrak{s l}_{n+1}}$ sending $\left(\lambda_{1}, \ldots, \lambda_{n}\right) \mapsto\left(\lambda_{1}, \ldots, \lambda_{n}, 0\right)$. We let $\mathcal{U}$ be the 2 -subcatgory given by 1-morphisms in this image, but with 2-morphisms calculated in $\mathcal{U}_{\mathfrak{s}_{I_{n+1}}}$. In terms of diagrams, this means we include bubbles (but not open strands) labeled by the root $\alpha_{n}$, and include the local relations we expect. The basis of Khovanov and Lauda given in [KL10] shows that for 1-morphisms $u$ and $v$, $\operatorname{Hom}_{\mathcal{U}}(u, v) \cong \operatorname{Hom}_{\mathcal{U}}(u, v) \otimes \mathbf{k}\left[\cup_{n}(1), \cup_{n}(2), \cdots\right]$ for the bubbles $\cup_{n}(k)$ with label $n$ and degree $k$ at the far left of the diagram. The result $\mathcal{U}$ is a 2-category with essentially the same structure as $\mathcal{U}$, but with slightly enlarged endomorphisms. In particular:

Proposition 3. For any indecomposable 1-morphism $u$ in $\mathcal{U}$, its image in $\mathcal{U}$ is also indecomposable.

Proof. By [Web15, 8.11], the algebra $\operatorname{End}_{\mathcal{U}}(u)$ is positively graded with only scalars in degree 0 . By the description above, $\operatorname{End}_{u}(u)$ has the same property, so $u$ is indecomposable in $\mathcal{U}$ as well.

2.2. The flag category and the Schur algebra. In this subsection, we'll discuss the corresponding story for the Schur algebra. This situation is more complicated, in that the same categorification of the Schur algebra has appeared in several guises at different points in the literature, and some explanation is required to explain how they are connected.

Fix an integer $d$. Throughout, we let $G=\mathrm{GL}_{d}(\mathbb{C})$, and $T$ be its diagonal subgroup. For each increasing $n-1$-tuple $0 \leq d_{1} \leq d_{2} \leq \cdots \leq d_{n-1} \leq d$, we have a corresponding flag variety $\mathrm{Fl}(\mathbf{d})$ with an action of $G$. Following their notation, let $H_{\mathbf{d}}:=H^{*}(\mathrm{Fl}(\mathbf{d}) ; \mathbf{k})$. We will also at times want to consider the the equivariant cohomology ring $H_{d}^{G}:=$ 
Comparison of canonical bases for Schur and universal enveloping algebras

$H_{G}^{*}(\mathrm{Fl}(\mathbf{d}) ; \mathbf{k})$. We let $\mathbf{d}^{\prime}=0 \leq d_{1} \leq \cdots \leq d_{i-1} \leq d_{i}+1 \leq \cdots \leq d_{n-1}$ and $\mathbf{d}^{i}=0 \leq d_{1} \leq \cdots \leq$ $d_{i} \leq d_{i}+1 \leq \cdots \leq d_{n-1}$. The cohomology ring $H_{\mathbf{d}^{i}}$ contains $H_{\mathbf{d}}$ and $H_{\mathbf{d}^{\prime}}$ as subrings by pullback. Let $H_{\mathbf{d}^{+i}}$ denote the bimodule where $H_{\mathbf{d}^{\prime}}$ acts on the left and $H_{\mathbf{d}}$ on the right, and $H_{\mathbf{d}^{-i}}$ the bimodule where we reverse these actions.

Definition 4. The flag category $\mathrm{Flag}_{d}$ is the 2-category given by:

- an object is an (n-1)-tuple $\mathbf{d}$ (note: in [KL10], the objects are defined to be the rings themselves. This is a distinction without a difference).

- a 1-morphism $\mathbf{d} \rightarrow \mathbf{d}^{\prime}$ is an object in the subcategory of $H_{\mathbf{d}^{\prime}}-H_{\mathbf{d}^{-}}$-bimodules generated by bimodules of the form $\mathrm{H}_{\mathbf{d}^{ \pm i}}$ under tensor, degree shift, direct sum and taking of summands.

- a 2-morphism is a degree preserving bimodule map.

The equivariant flag category $\mathrm{Flag}_{d}^{G}$ is the corresponding category with bimodules over $H_{\mathrm{d}}^{G}$.

Note that Flag ${ }_{d}$ is equipped with a duality induced by the Frobenius structure on $H_{\mathbf{d}}$ for each $\mathbf{d}$.

We can associate the $\mathfrak{s l}_{n}$-weight $\mu_{\mathbf{d}}=\left(d_{1}, d_{2}-d_{1}, d_{3}-d_{2}, \ldots, d_{n}-d_{n-1}, d-d_{n}\right)$ to each increasing $(n-1)$-tuple $\mathbf{d}$. The increasing property precisely guarantees that all the entries of this weight are non-negative. Having fixed $d$, there is at most one $\mathbf{d}$ such that $\mu=\mu_{\mathrm{d}}$, so we can also index flag varieties and cohomology rings with the weight $\mu_{\mathrm{d}}$ instead. In [KL10, §6] Khovanov and Lauda construct a functor $\Phi^{\prime}: \mathcal{U} \rightarrow$ Flag $_{d}$, which sends $\mu \mapsto \mathbf{d}$ if $\mu=\mu_{\mathbf{d}}$ and 0 otherwise. This functor sends

$$
\mathcal{E}_{i} \mapsto H_{\mathbf{d}^{+i}}\left(d_{i+1}-d_{i}-1\right) \quad \mathcal{F}_{i} \mapsto H_{\mathbf{d}^{-i}}\left(d_{i}-d_{i-1}\right) .
$$

Here $M(a)$ denotes the graded module $M$ with its grading shifted downward. Note that we have used a slightly different grading shift from [KL10, §6] which matches better with the geometry; note that the effect is just a shift by a fixed degree on all bimodules for $\mathbf{d}$ and $\mathbf{d}^{\prime}$ fixed.

This representation is compatible with the inclusion $\mathcal{U}_{\mathfrak{s I}_{n}} \rightarrow \mathcal{U}_{\mathrm{sI}_{n+1}}$ in the sense that a weight of the form $\left(\lambda_{1}, \ldots, \lambda_{n}, 0\right)$ will give an $n$-step flag where the last term must be the whole space for dimension reasons. Thus, the underlying cohomology ring is unchanged by this inclusion, as are all the bimodules attached to the Chevalley generators of $\mathcal{U}_{\mathfrak{s i n}_{n}}$. Thus, this inclusion allows us to extend the representation $\Phi^{\prime}$ to a representation $\Phi$ of $\mathcal{U}$.

There is a second, more geometric interpretation of this category we should also discuss. Let $\mathrm{X}=\sqcup_{\mu, v} \operatorname{Fl}(\mu) \times \mathrm{Fl}(v)$; as with any product of a space with itself, there is a convolution product of the constructible derived category of $X$ which endows this space with a monoidal structure. We wish to consider the subcategory of parity sheaves smooth along the G-orbits, as defined by Juteau, Mautner and Williamson [JMW14]. This is a monoidal subcategory as shown in [JMW14, 4.8]. If $\mathbf{k}$ is characteristic 0 , then parity sheaves are the same as sums of shifts of simple perverse sheaves smooth along the G-orbits, in which case this follows from the decomposition theorem of Beilinson, Bernstein and Deligne. In 2-category language:

Definition 5. Let Perv ${ }_{d}$ be the 2-category where

- objects are weights $\mu$. 
- 1-morphisms $v \rightarrow \mu$ are parity sheaves over $\mathbf{k}$ on $\mathrm{Fl}(\mu) \times \mathrm{Fl}(v)$ which are smooth along the G-orbits for the diagonal action. The composition of 1-morphisms

$$
\operatorname{Hom}(\mu, v) \times \operatorname{Hom}(v, \xi) \rightarrow \operatorname{Hom}(\mu, \xi)
$$

is given by convolution

$$
\mathscr{G} \star \mathscr{H}:=\left(p_{13}\right)_{*}\left(p_{12}^{*} \mathscr{G} \otimes_{\mathbf{k}} p_{23}^{*} \mathscr{H}\right)[\operatorname{dim} \mathrm{Fl}(\mu)+\operatorname{dim} \mathrm{Fl}(\xi)] .
$$

- 2-morphisms are morphisms in the constructible derived category.

We think of this as a graded 2-category where grading shift is the translation [1] in the derived category. A 2-morphism of degree $m$ is thus an element of $\mathrm{Ext}^{\mathrm{m}}$.

There is also an equivariant version Perv $\mathrm{P}_{d}^{G}$ of this category, where we consider G-equivariant parity sheaves. We'll use the notation $\operatorname{Perv}_{d}^{(G)}$ to indicate either one of these categories.

This 2-category also has an accompanying duality, induced by Verdier duality on parity sheaves.

While these categories seem very different in nature, they are actually very similar. The category of bimodules Flag ${ }_{d}$ is (to quote Soergel) the "poor man's version" of Perv $_{d}$, following similar results of Soergel for the complete flag variety [Soe90, Soe00]. Both Theorem 6 and Lemma 8 seem to be "well-known" in the correct circles, but the author does not believe they have appeared in the literature in this generality previously.

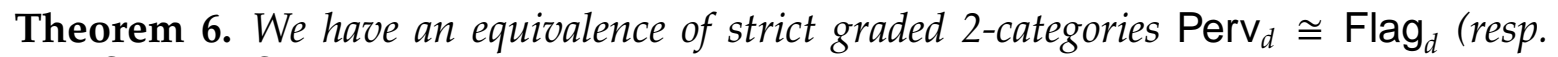
$\operatorname{Perv}_{d}^{G} \cong \mathrm{Flag}_{d}^{G}$ ) via the 2-functor

$$
\mathscr{G}: v \rightarrow \mu \mapsto H_{(G)}^{\bullet}(\operatorname{Fl}(\mu) \times \mathrm{Fl}(v) ; \mathscr{G})
$$

compatible with dualities.

Proof. The essential point is that Flag $_{d}$ and the cohomology of parity sheaves in $\mathrm{Fl}(\mu) \times \mathrm{Fl}(v)$ are both descriptions of singular Soergel bimodules modulo the positive degree elements in the ring of symmetric polynomials $R=H^{\bullet}(B G)=\mathbf{k}\left[x_{1}, \ldots, x_{d}\right]_{d}^{S_{d}}$. The category Flag $_{d}^{G}$ and equivariant cohomology correspond to Soergel bimodules without this quotient. Since this case is so similar, we will only consider the nonequivariant case. We let $S_{\mu} \subset S_{d}$ be the subgroup of the symmetric group acting on $[1, n]$ which preserves the flag of subsets $\left\{1, \ldots, d_{1}\right\} \subset\left\{1, \ldots, d_{2}\right\} \subset \ldots \subset\left\{1, \ldots, d_{n}\right\}$ and let $R_{\mu}=\mathbf{k}\left[x_{1}, \ldots, x_{d}\right]^{S_{\mu}}=H_{G}^{*}(\mathrm{Fl}(\mu))$.

By definition, a singular Soergel bimodule is a sum of shifts of summands of a tensor product of the form

$$
R_{\mu_{1}} \otimes_{R_{v_{1}}} R_{\mu_{2}} \otimes_{R_{v_{2}}} \cdots \otimes_{R_{v_{k-1}}} R_{\mu_{k}}
$$

for weights with $S_{\mu_{1}} \subset S_{v_{1}} \supset S_{\mu_{2}} \subset \cdots \subset S_{v_{k-1}} \supset S_{\mu_{k}}$. The ring $R$ acts diagonally on these bimodules, and as left or right modules they are free. This shows that

$$
\left(M \otimes_{R_{v}} N\right) \otimes_{R} \mathbf{k} \cong\left(M \otimes_{R} \mathbf{k}\right) \otimes_{\bar{R}_{v}}\left(N \otimes_{R} \mathbf{k}\right)
$$


so Khovanov and Lauda's bimodules defined in [KL10, 5.6] are reductions of the form $M \otimes_{R} \mathbf{k}$ where $M$ is a singular Soergel bimodule. Furthermore,

$$
\operatorname{Hom}\left(\bar{B}, \bar{B}^{\prime}\right) \cong k \otimes_{R} \operatorname{Hom}\left(B, B^{\prime}\right),
$$

so we can use [Wil, 7.4.1] to compute the dimension of $\operatorname{Hom}\left(\bar{B}, \bar{B}^{\prime}\right)$.

It is not completely obvious that every bimodule of this form is one of Khovanov and Lauda's since they do not include all tensor products of bimodules as in (1); they will only consider triples $\mu_{i}, v_{i}, \mu_{i+1}$ where $s_{k}, s_{k+1} \notin S_{v_{i}}$ and

$$
S_{\mu_{i}}=\left\langle s_{k}, S_{v_{i}}\right\rangle, S_{\mu_{i+1}}=\left\langle s_{k+1}, S_{v_{i}}\right\rangle \text {. or } S_{\mu_{i}}=\left\langle s_{k+1}, S_{v_{i}}\right\rangle, S_{\mu_{i+1}}=\left\langle s_{k}, S_{v_{i}}\right\rangle .
$$

However, there is considerable redundancy in the description of singular Soergel bimodules given in (1). By [Wil, 5.4.2], it is only necessary to consider tensor products corresponding to a choice of "reduced translation sequence" (as defined in [Wil, §1.3]). In type A, a reduced translation sequence is exactly one as in (2). Thus, Flag contains the reduction of any singular Soergel bimodule; that is, the category would remain unchanged if we defined 1-morphisms to be $\bar{B}:=B \otimes_{R} \mathbf{k}$ for $B$ a singular Soergel module over $R_{\mu}$ and $R_{\mu^{\prime}}$.

Now, we need to establish that the functor $H^{\bullet}(\mathrm{Fl}(\mu) \times \mathrm{Fl}(v) ;-)$ is an equivalence of categories compatible with convolution. The proof of equivalence is essentially the same as that given by Soergel in [Soe00, 4.2.1], but in a slightly more general context. The category of parity sheaves is generated by the pushforward $f_{*} \mathbf{k}_{B S}$ from an arbitrary generalized Bott-Samelson BS by [JMW14, 4.6]. Thus, we need only prove full faithfulness and commutation with convolution on these sheaves. Faithfulness follows from the same argument as [Soe00, 3.2.6] (note that the argument in the paper is incorrect, and corrected in [Soe]]). On the other hand, using [BGS96, 3.4.1], for parity sheaves $\mathscr{F}, \mathscr{G}$, we have

$$
\operatorname{dim} \mathbb{R} \operatorname{Hom}(\mathscr{F}, \mathscr{G})=\sum_{x \in \mathbf{X}^{\mathrm{T} \times T}} \operatorname{dim} \mathscr{F}_{x} \cdot \operatorname{dim} \mathscr{G}_{x},
$$

where $\mathscr{F}_{x}$ is the stalk at the $T \times T$-fixed point $x$. Note that we are just taking total dimensions of the cohomology of these complexes; since they are concentrated in odd or even degrees, that's the same as the absolute value of Euler characteristic. Since these dimensions of the stalks $\mathscr{F}_{x}$ also give the multiplicities of $\Delta$ - and $\nabla$-flags on $H^{\bullet}(\mathscr{F})$, we have that

$$
\operatorname{dim} \mathbb{R} \operatorname{Hom}(\mathscr{F}, \mathscr{G})=\operatorname{dim} \operatorname{Hom}\left(H^{\bullet}(\mathscr{F}), H^{\bullet}(\mathscr{G})\right)
$$

by [Wil, 7.4.1]. Thus, we also have fullness.

Commutation with convolution follows from the compatibility between the hypercohomology functor and pullback and pushforward between partial flag varieties. Using the notation of [JMW14], we consider the projection $\pi_{\mathbf{d}^{i}}^{\mathrm{d}^{\prime}}: \operatorname{Fl}\left(\mathbf{d}^{i}\right) \rightarrow \operatorname{Fl}(\mathbf{d})$. We have $H^{\bullet}\left(\left(\pi_{\mathbf{d}^{i}}^{\mathrm{d}^{\prime}} \times \mathrm{id}\right)_{*}\left(\pi_{\mathrm{d}^{i}}^{\mathrm{d}} \times \mathrm{id}\right)^{*} \mathscr{F}\right) \cong H_{\mathrm{d}^{+i}} \otimes_{H_{\mathrm{d}}} H^{\bullet}(\mathscr{F})$ by [WW08, 3.3]. Applying this inductively, we have an isomorphism

$$
H^{\bullet}\left(\left(f_{1}\right)_{*} \mathbf{k}_{B S_{1}} \star\left(f_{2}\right)_{*} \mathbf{k}_{B S_{2}}\right) \cong H^{\bullet}\left(f_{*} \mathbf{k}_{B S}\right) \cong \bar{B}_{B S} \cong \bar{B}_{B S_{1}} \otimes \bar{B}_{B S_{2}}
$$


where $B S$ is the Bott-Samelson for the concatenation of the translation sequences giving $B S_{1}$ and $B S_{2}$. Thus, hypercohomology is a 2-functor and thus a 2-equivalence.

Thus, this equivalence allows us to define a 2-functor $\Phi_{\mathrm{P}}^{(G)}: \mathcal{U} \rightarrow \operatorname{Perv}_{d}^{(G)}$, which sends

$$
\mathcal{E}_{i} \mapsto\left(\pi_{\mathbf{d}^{\prime}} \times \pi_{\mathbf{d}}\right)_{*} \mathbf{k}_{\mathbf{d}^{i}}\left[d_{i+1}-d_{i}-1\right] \quad \mathcal{F}_{i} \mapsto\left(\pi_{\mathbf{d}} \times \pi_{\mathbf{d}^{\prime}}\right)_{*} \mathbf{k}_{\mathbf{d}^{i}}\left[d_{i}-d_{i-1}\right] .
$$

One can think of this as a categorification of the BLM construction. By standard techniques in étale cohomology, if we let $X_{\mathbb{Z}}$ be the canonical integral form of $X$, the category $\operatorname{Perv}_{d}^{(G)}$ can be defined using $X_{K}$ for any field $K$, and will not depend on the underlying field, as long as we fix $\mathbf{k}$. Taking $\mathbf{k}=\mathbb{Q}_{\ell}, K=\overline{\mathbb{F}}_{p}$ for $\ell$ and $p$ distinct primes, the sheaves in Perv ${ }_{d}$ have canonical mixed structures of weight 0.

Attached to a mixed sheaf on $X_{\overline{\mathbb{F}}_{p}}$, we have a corresponding function on the rational points $X\left(\mathbb{F}_{p}\right)$ given by the trace of Frobenius. This defines a map $\tau: K\left(\operatorname{Perv}_{d}^{(G)}\right) \rightarrow$ $\mathbb{C}\left[\mathrm{X}\left(\mathbb{F}_{p}\right)\right]$. There is also a BLM map $\mathcal{B L M}: S_{p}(d, n) \rightarrow \mathbb{C}\left[\mathrm{X}\left(\mathbb{F}_{p}\right)\right]$ which sends

$$
E_{i} \mapsto\left\{\begin{array} { l l } 
{ p ^ { - d _ { i + 1 } + d _ { i } + 1 } } & { x \in \operatorname { i m } ( \pi _ { \mathbf { d } ^ { \prime } } \times \pi _ { \mathrm { d } } ) } \\
{ 0 } & { x \notin \operatorname { i m } ( \pi _ { \mathbf { d } ^ { \prime } } \times \pi _ { \mathbf { d } } ) }
\end{array} \quad F _ { i } \mapsto \left\{\begin{array}{ll}
p^{-d_{i}+d_{i-1}} & x \in \operatorname{im}\left(\pi_{\mathbf{d}} \times \pi_{\mathrm{d}^{\prime}}\right) \\
0 & x \notin \operatorname{im}\left(\pi_{\mathbf{d}} \times \pi_{\mathbf{d}^{\prime}}\right)
\end{array}\right.\right.
$$

Lemma 7. The map $\tau$ is an algebra map, with

$$
\tau\left(\left[\Phi_{\mathrm{P}}\left(\mathcal{E}_{i}\right)\right]\right)=\operatorname{B\mathcal {M}}\left(E_{i}\right) \quad \tau\left(\left[\Phi_{\mathrm{P}}\left(\mathcal{F}_{i}\right)\right]\right)=\operatorname{BLM}\left(F_{i}\right) .
$$

Proof. The Grothendieck trace formula implies that this map $\tau$ sends convolution of sheaves to convolution of functions, so that shows that $\tau$ is an algebra map. Also, by definition it sends the classes of the sheaves $\left(\pi_{\mathbf{d}^{\prime}} \times \pi_{\mathbf{d}}\right)_{*} \mathbf{k}_{\mathbf{d}^{i}}\left[d_{i+1}-d_{i}-1\right]$ and $\left(\pi_{\mathbf{d}} \times \pi_{\mathbf{d}^{\prime}}\right)_{*} \mathbf{k}_{\mathbf{d}^{i}}\left[d_{i}-d_{i-1}\right]$ to the functions of (3); ; the powers of $p$ appear because we must twist the mixed structure to remain pure of weight 0 .

Lemma 8. The Grothendieck group $K\left(\operatorname{Perv}_{d}^{(G)}\right) \cong K\left(\mathrm{Flag}_{d}^{(G)}\right)$ is isomorphic to the q-Schur algebra. If $\mathbf{k}$ is of characteristic 0 , then the classes of self-dual indecomposable objects correspond to the canonical basis.

Proof. First, by necessity, we have a map $K(\Phi): \dot{\mathbf{U}} \rightarrow K\left(\operatorname{Perv}_{d}^{(G)}\right)$ induced by the functor $\Phi$. The latter is spanned by the classes $[\mathcal{E}(O)]$ of the unique indecomposable parity sheaf $\mathcal{E}(O)$ with support equal to the orbit closure $\bar{O}$ by [JMW14, 4.6]. As shown in the proof of [JMW14, 4.6], there is a reduced translation sequence such that the pushforward from the Bott-Samelson in Perv ${ }_{d}$ is supported on $\bar{O}$ and contains $\mathcal{E}(O)$ as a summand with multiplicity 1 . The class of the pushforward is in the image of $K(\Phi)$ by definition, and we can assume all summands other than $\mathcal{E}(O)$ are by induction on the dimension of $O$. Thus, the map $\dot{\mathbf{U}} \rightarrow K\left(\operatorname{Perv}_{d}^{(G)}\right)$ is surjective.

On the other hand, the kernel of $K\left(\Phi^{(G)}\right)$ contains $1_{\mu}$ if $\mathrm{Fl}(\mu)=\emptyset$. Thus, $K\left(\Phi^{(G)}\right)$ factors through the $q$-Schur algebra, and induces a surjective map $S_{q}(d, n) \rightarrow K\left(\operatorname{Perv}_{d}^{(G)}\right)$. The dimension of these algebras coincide, since both are equal to the number of $G$-orbits on X. Thus, the map is an isomorphism. 
By Lemma 7, this isomorphism intertwines the maps $\tau$ and $\mathcal{B L M}$. If $\mathbf{k}$ has characteristic 0 , then the indecomposable parity sheaves on $\mathrm{X}$ are the simple perverse sheaves. Thus $\tau$ applied to one of these classes is the supertraces of Frobenius on the stalks of the IC sheaf. This is the same as the image under the map $\mathcal{B L M}$ of the canonical basis, by definition. This completes the proof.

Thus, as claimed in the introduction, the category Flag $_{d}^{(G)}$ or Perv $v_{d}^{(G)}$ provides a categorification of $S_{q}(d, n)$. Note that it if $\mathbf{k}$ has characteristic $p>0$, the indecomposables define a $p$-canonical basis $\dot{\mathbf{B}}_{d}^{(p)}$, which agrees with the canonical basis for $p$ large. We note that this is compatible with the $p$-canonical basis on the Hecke algebra defined by classes of parity sheaves on $\mathrm{Fl}(1,2,3, \ldots, n-1)$ (see [JMW14, 1.3.4]).

\section{Fullness}

The main result of this note is that the functor $\Phi_{\mathrm{P}}^{(G)}$ is "surjective" in an appropriate sense.

Theorem 9. The functor $\Phi_{\mathrm{P}}^{(G)}: \mathcal{U} \rightarrow \operatorname{Perv}_{d}^{(G)}$ is essentially surjective on 1-morphisms and full on 2-morphisms (that is, locally full). That is, for any 1-morphisms $u \rightarrow v$, we have an induced surjection $\operatorname{Hom}_{u}(u, v) \rightarrow \operatorname{Hom}_{\mathrm{Perv}_{d}^{(G)}}\left(\Phi_{\mathrm{P}}^{(G)} u, \Phi_{\mathrm{P}}^{(G)} v\right)$.

If $n$ is invertible in $\mathbf{k}$, then the restriction of $\Phi_{\mathrm{P}}$ to $\mathcal{U}$ has the same properties, but the restriction of $\Phi_{\mathrm{P}}^{G}$ does not.

We hope the statement of this theorem illustrates why we need to use $\mathcal{U}$ instead of $\mathcal{U}$; while $\mathcal{U}$ will suffice over a field of large enough characteristic, $\mathcal{U}$ allows us to make a cleaner statement covering the equivariant case as well. Note that it seems the characteristic requirement seems to be necessary; in [BL14], the authors must assume 2 is invertible to get the $\mathfrak{s l}_{2}$ version of this result.

Lemma 10. Let $A$ and $B$ be $\mathbf{k}$-linear 2-categories such that 2-morphism spaces are always finite dimensional. Any 2-functor $\Phi: A \rightarrow B$ which is essentially surjective on 1-morphisms and full on 2-morphisms induces a surjective map $K(\Phi): K(A) \rightarrow K(B)$ that sends the classes in $K(A)$ of indecomposable objects not in the kernel of $K(\Phi)$ to the classes of indecomposable objects in $K(B)$.

Proof. A full functor induces a surjection $\operatorname{End}(X) / J(X) \rightarrow \operatorname{End}(\Phi X) / J(\Phi X)$ on the quotients of endomorphism rings by Jacobson radical. Thus, if $X$ is indecomposable, $\operatorname{End}(X) / J(X)$ is a division algebra, and $\operatorname{End}(\Phi X) / J(\Phi X)$ must be a division algebra again or 0 . This shows that $\Phi X$ is indecomposable or 0 .

If $X$ and $Y$ are non-isomorphic indecomposables, $\operatorname{End}(\Phi(X \oplus Y)) / J(\Phi(X \oplus Y))$ must be 0 , a division algebra, or the sum of two division algebras. It cannot contain a $2 \times 2$ matrix algebra over either of these division algebras for dimension reasons, and thus we must have $\Phi X ¥ \Phi Y$ unless both are 0 .

Applying this result to the functor $\Phi_{\mathrm{P}}$ immediately gives a proof of Theorem $\mathrm{A}$, In fact, it gives us a stronger result, which extends this statement to $p$-canonical bases:

Theorem 11. The map $\phi$ induces a bijection between $\mathbf{B}^{(\mathbf{p})} \backslash\left(\dot{\mathbf{B}}^{(p)} \cap \operatorname{ker} \phi\right)$ and $\dot{\mathbf{B}}_{d}^{(p)}$. 
We'll give two proofs of Theorem 9 . This may seem redundant but both have interesting generalizations in different directions, and thus we think both are worth including.

Proof \# 1. Since the natural functor Perv $v_{d}^{G} \rightarrow \operatorname{Perv}_{d}$ is surjective, let us consider $\operatorname{Perv}_{d}^{G}$ first. We order the dimension functions $\mathbf{d}_{i}$ by pointwise comparison, and induct downward in this order. This is the same as the usual order on the weight lattice generated by $\mu-\alpha_{i}<\mu$.

We have that $\operatorname{Fl}(d, d, \ldots, d)$ is a point, so the only non-trivial 1-morphism is the identity, and its endomorphisms are just the scalars. In this case, fullness is clear. This establishes the base case.

Assume that we know the theorem for 1-morphisms $\mu^{\prime} \rightarrow v^{\prime}$ where either $\mu^{\prime}>\mu$ or $v^{\prime}>v$. Assume that $u$ and $v$ are indecomposable. Recall that $\mathcal{U}$ has a "triangular decomposition" into two subcategories $\mathcal{U}^{+}$and $\mathcal{U}^{-}$generated by the $\mathcal{E}_{i}{ }^{\prime} \mathrm{s}$ and $\mathcal{F}_{i}{ }^{\prime} \mathrm{s}$ respectively. We now prove two smaller claims:

(1) if $v$ is not in the image of $\mathcal{U}^{-}$, then $\operatorname{Hom}_{u}(u, v) \rightarrow \operatorname{Hom}_{\operatorname{Perv}_{d}^{G}}\left(\Phi_{\mathrm{P}}^{G} u, \Phi_{\mathrm{P}}^{G} v\right)$.

(2) if $u$ is not in the image of $\mathcal{u}^{+}$, then $\operatorname{Hom}_{u}(u, v) \rightarrow \operatorname{Hom}_{\operatorname{Perv}_{d}^{G}}\left(\Phi_{\mathrm{P}}^{G} u, \Phi_{\mathrm{P}}^{G} v\right)$.

Let us first consider (1). If $v$ is not in the image of $\mathcal{U}^{-}$then by [Web15, 5.12], we have that $v$ is a summand of $\mathcal{E}_{i} v^{\prime}$ for some 1-morphism $\mu+\alpha_{i} \rightarrow v$; let $e: \mathcal{E}_{i} v^{\prime} \rightarrow \mathcal{E}_{i} v^{\prime}$ by an idempotent whose image is $v$, and $v^{\prime \prime}$ be the image of $1-e$, that is the complementary summand. By assumption, we have a surjection

$$
\operatorname{Hom}_{u}\left(u, \mathcal{E}_{i} v^{\prime}\right) \cong \operatorname{Hom}_{u}\left(\mathcal{F}_{i} u, v^{\prime}\right) \rightarrow \operatorname{Hom}_{\mathrm{Perv}_{d}^{G}}\left(\mathcal{F}_{i} \Phi_{\mathrm{P}}^{G} u, \Phi_{\mathrm{P}}^{G}{v^{\prime}}^{\prime}\right) \cong \operatorname{Hom}_{\mathrm{Perv}}^{G}\left(\Phi_{\mathrm{P}}^{G} u, \mathcal{E}_{i} \Phi_{\mathrm{P}}^{G}{v^{\prime}}^{\prime}\right)
$$

With we compose this map with the idempotent $\Phi_{P}^{G} e$, then we obtain a surjection $\operatorname{Hom}_{u}\left(u, \varepsilon_{i} v^{\prime}\right) \rightarrow \operatorname{Hom}_{\mathrm{Perv}_{d}^{G}}\left(\Phi_{\mathrm{P}}^{G} u, \Phi_{\mathrm{P}}^{G} v\right)$, which kills $\operatorname{Hom}_{u}\left(u, v^{\prime \prime}\right)$; thus, the induced map $\operatorname{Hom}_{u}\left(u, \mathcal{E}_{i} v^{\prime} \operatorname{Hom}_{\operatorname{Perv}_{d}^{G}}\left(\Phi_{\mathrm{P}}^{G} u, \Phi_{\mathrm{P}}^{G}\right)\right.$ is surjective as desired. Claim (2) follows by a symmetric argument.

Thus, it remains to establish $\operatorname{Hom}_{u}(u, v) \rightarrow \operatorname{Hom}_{\mathrm{Perv}}^{G}\left(\Phi_{\mathrm{P}}^{G} u, \Phi_{\mathrm{P}}^{G} v\right)$ for $u$ in the image of $\mathcal{U}^{-}$and $v$ in the image of $\mathcal{U}^{+}$. For reasons of weight, the target can only be non-zero if $\mu=v$ and $u=v=\mathrm{id}_{\mu}$. Thus, we must prove that

$$
\operatorname{Hom}_{\mathcal{U}}\left(\mathrm{id}_{\mu}, \mathrm{id}_{\mu}\right) \rightarrow \operatorname{Hom}_{\text {Perv }_{d}^{G}}\left(\operatorname{id}_{\mu}, \operatorname{id}_{\mu}\right) \cong H_{G}^{*}(\operatorname{Fl}(\mu)) \rightarrow \operatorname{Hom}_{\text {Perv }_{d}^{G}}\left(\operatorname{id}_{\mu}, \operatorname{id}_{\mu}\right) \cong H^{*}(\operatorname{Fl}(\mu)) \text {. }
$$

Recall that $H_{G}^{*}(\mathrm{Fl}(\mu)) \cong R_{\mu}$ is generated by the Chern classes $c_{k}\left(T_{i}\right)=e_{k}\left(x_{d^{i-1}+1}, \ldots, x_{d^{i}}\right)$ for the tautological bundles $T_{i}=W^{i} / W^{i-1}$ on $\mathrm{Fl}(\mu)$ modulo the geometric relations that $c_{k}\left(T_{i}\right)=0$ if $k>\operatorname{rk} T_{i}=d_{i}-d_{i-1}$. By [KL10, (6.47)], the image of $\operatorname{Hom}_{u}\left(\operatorname{id}_{\mu}, \mathrm{id}_{\mu}\right)$ is the algebra generated by the coefficients of $c\left(T_{i+1}\right) / c\left(T_{i}\right)$. Extending to $\mathcal{U}$ gives $c\left(T_{n}\right)$ as the image of bubbles with label $n$. Thus the coefficients of $c\left(T_{i}\right)$ for each $i$ lie in this image, and they generate.

Now consider working non-equivariantly in Perv ${ }_{d}$. To complete the proof of the theorem, we need to show that if $n$ is invertible, then $\operatorname{Hom}_{\mathcal{U}}\left(\mathrm{id}_{\mu}, \mathrm{id}_{\mu}\right)$ surjects to Homperv $_{d}\left(\operatorname{id}_{\mu}, \mathrm{id}_{\mu}\right) \cong H^{*}(\operatorname{Fl}(\mu)) \cong \bar{R}_{\mu}$. The ring $\bar{R}_{\mu}$ is isomorphic to the quotient of $R_{\mu}$ with the additional relation that $c\left(T_{1}\right) \cdots c\left(T_{n}\right)=1$. In this case, the image of 
$\operatorname{Hom}_{\mathcal{U}}\left(\mathrm{id}_{\mu}, \mathrm{id}_{\mu}\right)$ contains

$$
c\left(T_{k}\right)^{n}=c\left(T_{1}\right) \cdots c\left(T_{n}\right) \prod_{i=1}^{k-1}\left(c\left(T_{i+1}\right) / c\left(T_{i}\right)\right)^{i} \prod_{i=k}^{n-1}\left(c\left(T_{i}\right) / c\left(T_{i+1}\right)\right)^{n-i} .
$$

Thus, if $n$ is invertible then the fractional binomial coefficents $\left(\begin{array}{c}1 / n \\ m\end{array}\right)$ exist and we can take the $n$th root of this power series, and thus obtain $c\left(T_{k}\right)$. This shows the surjectivity.

Remark 12. While we avoided using these results for the sake of clarity, this argument essentially uses Rouquier's Jordan-Hölder decomposition [Rou, 5.8] of a categorical module for Perv ${ }_{d}$, with its action of $\mathfrak{s l}_{n} \times \mathfrak{s l}_{n}$ on the left and right. The category Perv ${ }_{d}$ has a filtration whose successive quotients are categorifications of simple modules for $\mathfrak{s l}_{n} \times \mathfrak{s l}_{n}$. These quotients are generated by the image of $\mathrm{id}_{\mu}$ modulo the subcategory generated by weights $>\mu$. Thus, we can reduce to showing fullness on $\mathrm{id}_{\mu}$ by [Rou, 5.4], and complete the proof as above.

Proof \#2. As in Proof \# 1, we'll only consider Perv ${ }_{d}^{G}$. In this proof, we'll use an opposite reduction. Instead of relying on the highest weight vectors, we concentrate on the special weight $(1, \ldots, 1)$ when $d=n$. The corresponding flag variety is the complete flag variety $\mathrm{Fl}(1,2, \ldots, n-1)$, and the corresponding bimodules are honest Soergel bimodules. In this case, we can exploit the work of Elias and Khovanov which gives a diagrammatic description of this category.

We note that we can always increase $n$ by adding a redundant step in the flag, or decrease $n$ by removing a step where $d^{i}=d^{i+1}$. If $d^{j}<j$, then we must have a redundancy we can remove to decrease $j$; thus, we may assume that $d^{j} \geq j$ for all $j$. On the other hand, if $d^{j}>j$, we can add redundant flags until $d^{j}=d^{j+1}=\cdots=d^{d^{j}}$. Necessarily, the next step of the flag must be of higher dimension. If we think of this as a weight of $\mathfrak{s l}_{n}$, it is a positive integer $a_{1}$ followed by $a_{1}-1$ zeros, then $a_{2}$ followed by $a_{2}-1$ zeros, etc. Thus, we may assume that $n=d$ and our weight is of this form.

We now use the claim:

(1) every morphism $\mu \rightarrow v$ between two weights of the form above is a summand of a composition factoring through the weight $(1, \ldots, 1)$.

The principle we use here is very simple: if $\mu$ is a weight with $\alpha_{i}^{\vee}(\mu)>0$, then the identity functor is a summand of $\mathcal{E}_{i} \mathcal{F}_{i}$, by the $\mathfrak{s l}_{2}$ relations. It will also considerably simplify our computation if we note that when $\varepsilon_{i}$ acts trivially on this weight space (as is the case if $d^{i-1}=d^{i}$ ), then $\mathcal{E}_{i} \mathcal{F}_{i} \cong \mathrm{id}^{\alpha_{i}^{\vee}(\mu)}$. Thus, if $a_{i}=2$ in one of the examples above, we have that on the $\mu$-weight category:

$$
\mathcal{E}_{d^{i}+1} \mathcal{F}_{d^{i}+1} \cong \mathrm{id}^{\oplus 2} \oplus \mathcal{F}_{d^{i}+1} \mathcal{E}_{d^{i}+1}
$$

similarly, if $a_{i}=3$, then

$$
\mathcal{E}_{d^{i}+1} \varepsilon_{d^{i}+2} \varepsilon_{d^{i}+1} \mathcal{F}_{d^{i}+1} \mathcal{F}_{d^{i}+2} \mathcal{F}_{d^{i}+1} \cong \mathcal{E}_{d^{i}+1} \varepsilon_{d^{i}+2} \mathcal{F}_{d^{i}+2} \mathcal{F}_{d^{i}+1}^{\oplus 2} \cong \mathcal{E}_{d^{i}+1} \mathcal{F}_{d^{i}+1}^{\oplus 2} \cong \mathrm{id}^{\oplus 6}
$$


Thus, if our weight is of the form described above, applying this fact inductively, we find that whenever $d_{j}=j$, we have that

$$
\begin{aligned}
\mathrm{id}^{\left(d_{j+1}-d_{j}\right) !} \cong \mathcal{E}_{d^{j+1}} \mathcal{E}_{d^{j}+2} \cdots \mathcal{E}_{d^{j+1}} \mathcal{E}_{d^{j+1}} \varepsilon_{d^{j+2}} \cdots \mathcal{E}_{d^{j+1}-1} \cdots \mathcal{E}_{d^{i}+1} \mathcal{E}_{d^{i+2}+2} \mathcal{E}_{d^{i}+1} \\
\mathcal{F}_{d^{i}+1} \mathcal{F}_{d^{i}+2} \mathcal{F}_{d^{i}+1} \cdots \mathcal{F}_{d^{j+1}-1} \cdots \mathcal{F}_{d^{j}+2} \mathcal{F}_{d^{j}+1} \mathcal{F}_{d^{j+1}} \cdots \mathcal{F}_{d^{j}+2} \mathcal{F}_{d^{j+1}} .
\end{aligned}
$$

Aficionados of category $O$ will recognize this as the principle that translation off of and then back onto a wall is a multiple of the identity functor. Applying this for each $d_{j}=j$ gives the desired factorization.

Thus, it suffices to prove fullness for 1-morphisms of the form $u^{\prime} \circ u$ and $v \circ v^{\prime}$ factoring through $(1, \ldots, 1)$ (as we argued earlier, fullness is not harmed by replacing a module with one that it is a summand of). By the isomorphism

$$
\operatorname{Hom}\left(u^{\prime} \circ u, v \circ v^{\prime}\right) \cong \operatorname{Hom}\left(v^{L} \circ u, v^{\prime} \circ u^{R}\right)
$$

we can now assume that $\mu=v=(1, \ldots, 1)$. In this case, every indecomposable module is a summand of a 1-morphism factoring through a weight space killed by the map to the Schur algebra (in which case, the fullness is trivial) or it is a summand of a Bott-Samelson object $Z_{\mathbf{i}}:=\mathcal{E}_{i_{1}} \mathcal{F}_{i_{1}} \mathcal{E}_{i_{2}} \mathcal{F}_{i_{2}} \cdots \mathcal{E}_{i_{n}} \mathcal{F}_{i_{n}}$. The image $\Phi_{\mathrm{P}}^{G}\left(Z_{\mathbf{i}}\right)$ is a Bott-Samelson bimodule. The Hom spaces between these have been calculated by Elias and Khovanov [EK10]. Furthermore, in [MSV13, 6.5-6], Mackaay, Stošić and Vaz show the surjectivity of the map $\operatorname{Hom}_{u}(u, v) \rightarrow \operatorname{Hom}_{\operatorname{Perv}_{d}^{G}}\left(\Phi_{\mathrm{P}}^{G} u, \Phi_{\mathrm{P}}^{G} v\right)$ by giving diagrams in $\mathcal{U}$ which hit each of the Elias and Khovanov's generators. Thus, surjectivity follows.

This fact also has a significant consequence in the theory of categorification. Recall that Mackaay, Stošić and Vaz [MSV13] define a categorification $\mathcal{S}(n, d)$ of the $q$-Schur algebra as a subquotient of $\mathcal{U}$; one restricts objects to $\left(d_{1}, \ldots, d_{n}\right)$ where $\sum_{i} d_{i}=d$, and then sets to 0 any 1-morphism factoring through objects with any $d_{i}<0$. As before, it is really more convenient to use $\mathcal{U}$, and we let $\mathcal{S}(n, d)$ to the corresponding quotient of this category.

Proposition 13. The category $\mathcal{S}(n, d)$ is equivalent to $\operatorname{Perv}_{d}^{G}$.

We fully expect that as a quotient of $\mathcal{U}$, the 2-category $\mathcal{S}(n, d)$ is equivalent to the image of the functor $\Phi_{\mathrm{P}}: \mathcal{U} \rightarrow \operatorname{Perv}_{d}^{G}$; the proof below should work to show this, but is made a bit more difficult by the fact that the image of End $\mathcal{U}\left(\operatorname{id}_{\mu}\right)$ in $H_{\mathbf{d}}^{G}$ is not an especially well understood ring.

Proof. Since $\Phi_{\mathrm{P}}$ kills the correct weights, we have a functor $\Phi_{\S}: \mathcal{S}(n, d) \rightarrow \operatorname{Perv}_{d}^{G}$ which we need to show is injective. As suggested in Remark 12, we can show this using Rouquier's Jordan-Hölder filtration, or repeating the argument of Proof \# 1. In either case, we reduce to showing it suffices to check injectivity of the map

$$
\text { End }_{\delta(n, d)}\left(\operatorname{id}_{\mu}\right) \rightarrow \text { End }_{\text {Perv }_{d}^{G}}\left(\operatorname{id}_{\mu}\right) \text {. }
$$

Let $\mathbf{d}$ be the dimensions of the flag corresponding to $\mu$. The target space is simply $H_{\mathbf{d}}^{G}$, the space of symmetric polynomials for the permutation group $S_{\mathrm{d}}$. Thus, we need 
only show that the kernel of the map from $\operatorname{End}_{\mathcal{U}}\left(1_{\mu}, 1_{\mu}\right)$ to $\operatorname{End}_{\mathrm{Perv}_{d}^{G}}\left(1_{\mu}, 1_{\mu}\right)$ is spanned by classes which are 0 in $\mathcal{S}(n, d)$.

Let $B_{i}(u)=\sum u^{k} \cup_{i}(k)$ be the power series loaded with bubbles $\cup_{i}(k)$ with label $i$ and degree $k$. The image of $B_{i}(u)$ in $H_{\mathrm{d}}^{G}$ is $c\left(T_{i}\right) / c\left(T_{i+1}\right)$ with the convention that $T_{n+1}$ is the trivial bundle, so for each $i$, we have that $c\left(T_{i}\right)=C_{k}(u)=\prod_{k=i}^{n} B_{k}(u)$. Thus, we need to show that the vanishing of this product in degrees $>d_{i}-d_{i-1}$ is one of the relations of $\mathcal{S}(n, d)$. We can prove this by induction on $i$. If $i=n$, then the bubble $\bigcup_{i}(k)$ will vanish if it is not fake, which holds when $k>d-d_{n-1}$.

We consider $n-i+1$ concentric nested bubbles with labels $n, n-1, n-2, \ldots, i$ with thicknesses $d-d_{n}, d_{n}-d_{n-1}, \ldots, d_{i+1}-d_{i}, 1$, all counterclockwise except the innermost, and with the only dots being on the innermost. The outside region is labeled with the weight $\left(d_{1}, d_{2}-d_{1}, \ldots, d-d_{n}, 0\right)$. We use this to construct the power series

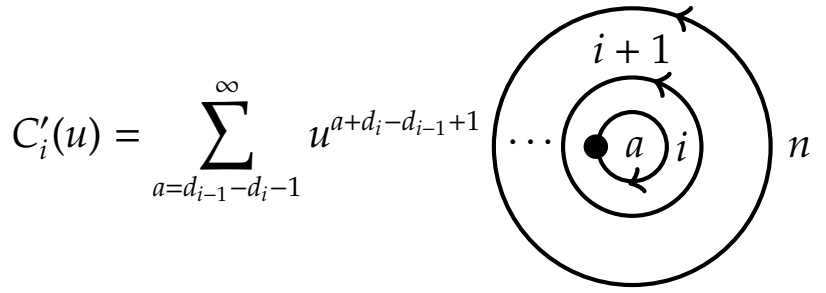

Note that the inner region has weight $\left(d_{1}, d_{2}-d_{1}, \ldots, d_{i}-d_{i-1}+1,-1, d_{i+1}-d_{i}, \ldots, d-d_{n}\right)$, so if the number of dots $a$ is non-negative, then this diagram vanishes in $\mathcal{S}(n, d)$.

The bubble slides show that sliding the innermost bubble out gives a gives that $C_{i}^{\prime}(u)$ is equal to $B_{i}(u)$ times a power series loaded with the $n-i$ remaining nested bubbles, with the innermost now decorated with the elementary symmetric function of degree $b$ times $u^{b}$. The result [KLMS12, 4.10] shows that this is the power series $C_{i+1}^{\prime}(u)$. Thus, we have that $C_{i}^{\prime}(u)=B_{i}(u) C_{i+1}^{\prime}(u)$, so by induction $C_{i}^{\prime}(u)=C_{i}(u)$.

As we noted before, this shows that the coefficients $C_{i}^{(a)}$ for $a>d_{i}-d_{i-1}$ vanish in $\mathcal{S}(n, d)$. This shows the injectivity of the map, and thus the proof is complete.

It's clear that we have a functor $\mathcal{S}(n, d+n) \cong \operatorname{Perv}_{d+n}^{G} \rightarrow \mathcal{S}(n, d) \cong \operatorname{Perv}_{d}^{G}$ which is induced by the functor $\mathcal{U} \rightarrow \mathcal{U}$ which sends $\left(\mu_{1}, \ldots, \mu_{n}\right) \rightarrow\left(\mu_{1}-1, \ldots, \mu_{n}-1\right)$ and is the identity on 1- and 2-morphisms. Thus, these categories form an inverse system as in [BL14, §3]. Combining Theorem 9 with the injectivity proven in [KL10, 6.16] shows that this local system has the same "partial graded locally full and faithful" property as shown in [BL14, 2.12]. This allows us to generalize their theorem [BL14, 3.2] with the same proof to show that:

Proposition 14. The 2-category $U$ is the inverse limit of the equivariant flag categories $\mathrm{Flag}_{d}^{G}$. If $n$ is invertible in $\mathbf{k}$ then $\mathcal{U}$ is the inverse limit of the categories $\mathrm{Flag}_{d}$.

Even if $n$ is not invertible, we can write $\mathcal{U}$ as the inverse limit of its image in $\mathrm{Flag}_{d}$ or Flag ${ }_{d}^{G}$.

\section{REFERENCES}

[BGS96] Alexander Beilinson, Victor Ginzburg, and Wolfgang Soergel, Koszul duality patterns in representation theory, J. Amer. Math. Soc. 9 (1996), no. 2, 473-527. 
[BL14] Anna Beliakova and Aaron D. Lauda, Categorified quantum $\mathfrak{s l}_{2}$ is an inverse limit of flag 2-categories, Transform. Groups 19 (2014), no. 1, 1-26. MR 3177365

[BLM90] A. A. Beilinson, G. Lusztig, and R. MacPherson, A geometric setting for the quantum deformation of $\mathrm{GL}_{n}$, Duke Math. J. 61 (1990), no. 2, 655-677. MR 1074310 (91m:17012)

[Bru] Jon Brundan, On the definition of Kac-Moody 2-category, arXiv:1501.00350.

[CL15] Sabin Cautis and Aaron D. Lauda, Implicit structure in 2-representations of quantum groups, Selecta Math. (N.S.) 21 (2015), no. 1, 201-244.

[EK10] Ben Elias and Mikhail Khovanov, Diagrammatics for Soergel categories, Int. J. Math. Math. Sci. (2010), Art. ID 978635, 58. MR 3095655

[JMW14] Daniel Juteau, Carl Mautner, and Geordie Williamson, Parity sheaves, 2014, pp. 1169-1212. MR 3230821

[KL10] Mikhail Khovanov and Aaron D. Lauda, A categorification of quantum sl( $n$ ), Quantum Topol. 1 (2010), no. 1, 1-92. MR 2628852 (2011g:17028)

[KLMS12] Mikhail Khovanov, Aaron D. Lauda, Marco Mackaay, and Marko Stošić, Extended graphical calculus for categorified quantum sl(2), Mem. Amer. Math. Soc. 219 (2012), no. 1029, vi+87. MR 2963085

[Lus93] George Lusztig, Introduction to quantum groups, Progress in Mathematics, vol. 110, Birkhäuser Boston Inc., Boston, MA, 1993.

[MSV13] Marco Mackaay, Marko Stošić, and Pedro Vaz, A diagrammatic categorification of the q-Schur algebra, Quantum Topol. 4 (2013), no. 1, 1-75. MR 2998837

[Rou] Raphael Rouquier, 2-Kac-Moody algebras, arXiv:0812.5023

[Soe] Wolfgang Soergel, Corrections for "On the relation between intersection cohomology and representation theory in positive characteristic", http://home.mathematik.uni-freiburg.de/soergel/PReprints/KorrICP.pdf

[Soe90] Kategorie O, perverse Garben und Moduln über den Koinvarianten zur Weylgruppe, J. Amer. Math. Soc. 3 (1990), no. 2, 421-445. MR MR1029692 (91e:17007)

[Soe00] On the relation between intersection cohomology and representation theory in positive characteristic, J. Pure Appl. Algebra 152 (2000), no. 1-3, 311-335, Commutative algebra, homological algebra and representation theory (Catania/Genoa/Rome, 1998). MR 1784005 (2001k:20098)

[SV00] O. Schiffmann and E. Vasserot, Geometric construction of the global base of the quantum modified algebra of $\widehat{\mathfrak{g l}}_{n}$, Transform. Groups 5 (2000), no. 4, 351-360. MR 1800532 (2001k:17029)

[Web] Ben Webster, Knot invariants and higher representation theory, arXiv:1309.3796

[Web15] _ Canonical bases and higher representation theory, Compos. Math. 151 (2015), no. 1, 121-166.

[Wil] Geordie Williamson, Singular Soergel bimodules,http://people.mpim-bonn.mpg.de/geordie/GW-thesis.pdf

[WW08] Ben Webster and Geordie Williamson, A geometric model for Hochschild homology of Soergel bimodules, Geom. Topol. 12 (2008), no. 2, 1243-1263. MR 2425548 (2009h:20050) 